\title{
Registered report: Investigating a preference for certainty in conversation among autistic adults compared to dyslexic adults and the general population
}

\author{
Alexander C Wilson ${ }^{\text {Corresp., }}{ }^{,}$, Dorothy V M Bishop ${ }^{1}$ \\ ${ }^{1}$ Department of Experimental Psychology, University of Oxford, Oxford, UK \\ Corresponding Author: Alexander C Wilson \\ Email address: alexander.wilson2@psy.ox.ac.uk
}

Social communication difficulties are a diagnostic feature in autism. These difficulties are sometimes attributed, at least in part, to impaired ability in making inferences about what other people mean. In this registered report, we test a competing hypothesis that the communication profile of adults on the autism spectrum can be more strongly characterised by reduced confidence in making inferences in the face of uncertain information. We will test this hypothesis by comparing the performance of 100 autistic and 100 non-autistic adults on a test of implied meaning, using a test of grammaticality judgements as a control task. We hypothesise that autistic adults will report substantially lower confidence, allowing for differences in accuracy, than non-autistic adults on the test of implied meaning compared to the grammaticality test. In addition, we hypothesise that reduced confidence in drawing inferences will relate to the cognitive trait Intolerance of Uncertainty and self-reported social communication challenges. Finally, we will conduct exploratory analysis to assess the specificity of the communication profile of the autistic adults by comparing their performance to that of dyslexic adults, who might also be expected to experience challenges with language and communication. 
1 Registered report: Investigating a preference for certainty in conversation among autistic

2

3

4

5

6

7

8

9

10

11

12

13

14

15

16

17

18 adults compared to dyslexic adults and the general population

WILSON, Alexander Connolly

BISHOP, Dorothy Vera Margaret

Department of Experimental Psychology, University of Oxford

12 Please address all correspondence to Alexander Wilson (alexander.wilson2@psy.ox.ac.uk). 


\section{Abstract} sometimes attributed, at least in part, to impaired ability in making inferences about what other people mean. In this registered report, we test a competing hypothesis that the communication

24 profile of adults on the autism spectrum can be more strongly characterised by reduced confidence in making inferences in the face of uncertain information. We will test this hypothesis by comparing the performance of 100 autistic and 100 non-autistic adults on a test of implied meaning, using a test of grammaticality judgements as a control task. We hypothesise that autistic adults will report substantially lower confidence, allowing for differences in accuracy, than non-autistic adults on the test of implied meaning compared to the grammaticality test. In addition, we hypothesise that reduced confidence in drawing inferences will relate to the cognitive trait Intolerance of Uncertainty and self-reported social communication challenges. Finally, we will conduct exploratory analysis to assess the specificity of the communication profile of the autistic adults by comparing their performance to that of dyslexic adults, who might also be expected to experience challenges with language and communication. 


\section{Introduction}

Persistent challenges with social communication are a defining feature for the diagnosis

of autism (American Psychiatric Association, 2013). The underlying nature of these challenges

44

remains unclear, although they are sometimes attributed to a core impairment in pragmatics (e.g. Baron-Cohen, 1988; Rapin \& Dunn, 2003). Pragmatics refers to the role of context in communication, including the ability to "read between the lines" to infer intended meaning beyond what is explicitly stated (Baird \& Norbury, 2016). However, empirical research suggests that pragmatic difficulties may be rather subtle in autistic people, and mostly attributable to language ability (Kalandadze, Norbury, Nærland \& Næss, 2018; Loukusa \& Moilanen, 2009). An alternative suggestion is that social communication difficulties are less the result of an impairment in pragmatics, but more impacted by cognitive preferences that differ between autistic and non-autistic people. We propose that a preference for certainty and explicit communication commonly occurs in autistic people, and that this trait may be a critical factor in the communication difficulties experienced by autistic people, as communicative situations often involve ambiguity, uncertainty and implied meanings.

\section{Intolerance of Uncertainty}

A "preference for certainty and explicit communication" may link to the widelyresearched cognitive trait, Intolerance of Uncertainty (IU). IU has been defined as a tendency to negatively evaluate uncertain situations and information (Shihata, McEvoy, Mullan \& Carleton, 
2016). We use the term "Intolerance of Uncertainty" in line with previous research and intend to convey a value-neutral meaning in using it, as we recognise that high levels of IU may be an understandable, even adaptive, response where individuals have experienced mishaps in confusing situations. IU has mostly been investigated as a transdiagnostic construct that plays a central role in emotional disorders across the general population (see Shihata et al., 2016 for a review), but it also seems especially relevant to autism, with autistic children and adults showing significantly elevated levels of the trait compared to the general population (e.g. Hwang et al., 2020; Vasa et al., 2018). IU has been closely linked to anxiety in autistic people (Jenkinson et al., 2020), and also relates to core features of autism, including social difficulties, sensory sensitivities, insistence on sameness and repetitive behaviours (e.g. Hwang et al., 2020; Vasa et al., 2018; Wigham et al., 2015).

A possible link between IU and communication in autistic people remains largely unexplored. However, there are reasons to believe that a link is plausible. First, inferential models of communication, such as Relevance Theory (Sperber \& Wilson, 1986), propose that communication inherently involves uncertainty. Under Relevance Theory, language comprehension is not simply a process of "understanding what the words mean", as there are often indeterminacies and ambiguities in uses of language; instead, the words are used as evidence by the listener in supporting a hypothesis about what the speaker probably means in the context, i.e. inferring intended meaning under uncertainty. Relevance Theory suggests that there is a gradient of uncertainty in communication. Sometimes, the listener can rely mostly on the explicit content of the utterance to compute the intended meaning, but in other situations there is a greater reliance on inferential processing to understand the speaker's probable meaning by integrating the utterance with contextual cues and world knowledge. Compare for instance the 
83 utterances "No, let's stay inside" and "It's quite cold today" as responses to a suggestion to go

84 outside. In the second example, the speaker communicates implicitly, leaving the listener to

85 process the implicature that they would probably prefer to stay inside. In a previous study, we

86 provided evidence for cognitive differences between autistic and non-autistic people in

87 processing implicatures (Wilson \& Bishop, 2020b). Crucially, it seemed that a cognitive

88 preference for certainty and explicit communication, and not simply reduced ability, may

89 account for some of the differences. Participants completed the Implicature Comprehension Test,

90 which required individuals to listen to short conversational interchanges that are followed by a

91 comprehension question to assess whether an implied meaning has been processed; test-takers

92 responded with “yes”, "no” or “don’t know”. Controlling for grammar/vocabulary ability, we

93 found that autistic adults $(N=66)$ were $6.19,95 \%$ CI $[3.63,10.39]$, times more likely to select

94 the "don't know" rather than the correct response compared to non-autistic people $(N=118)$, and

95 also $2.56,95 \%$ CI $[1.76,3.77]$, times more likely to choose the "incorrect" response (Wilson \&

96 Bishop, 2020b). Group differences were large, and performance on the test gave $76 \%$ sensitivity

97 and specificity for differentiating between autistic and non-autistic groups. On the face of it,

98 these results suggest that autistic people have difficulties inferring the gist of a speaker's

meaning, as predicted by the 'central coherence' theory, which proposes that autistic people may

100 show less tendency than non-autistic people to process information at a global level (Frith, 101 1989).

However, in an alternative version of the test without a "don't know" response, autistic individuals showed high accuracy for items for which they had selected "don't know" first time round. This marked tendency to select "don't know" when given a chance, but to process the inference as intended when constrained by the task, suggested reduced confidence in the face of 
106

107

108

109

110

111

112

113

114

115

116

117

118

119

120

121

122

124

125

126

127

128

uncertain information and a preference for explicit communication. This could be due to possible difficulties around metacognition in autistic people, who may experience a mismatch between performance and confidence in their performance due to differences in self-monitoring. There is evidence that autistic people may show such a mismatch (Grainger et al., 2016; Nicolson et al., 2019) although there is some concern about the replicability of these results (Maras et al., 2020). An alternative view would be that it is less an issue of metacognitive "ability", and more about differences in personality/cognitive preference, with the well-replicated elevated levels of IU in autistic people (e.g. Hwang et al., 2020; Vasa et al., 2018) accounting for this apparent preference for explicit communication observed in our previous study. In the present study, we aim to replicate this finding with more refined methods. In an adapted version of the Implicature Comprehension Test, individuals will respond using a 4-point scale of "yes", "maybe yes", "maybe no", and "no", allowing us to capture accuracy and confidence in the same measure. We hypothesise that confidence is likely to be affected specifically in a pragmatic language task (i.e. where the individual needs to make flexible context-dependent inference about uncertain implied meanings), and not on tasks focused on more structural, codified aspects of language such as grammatical competence. As such, we present the Grammaticality Decision Test as a control task with a similar response format to the pragmatic task to test the specificity of any differences.

We propose that reduced confidence on the Implicature Comprehension Test will be a marker of IU in autistic people, and may be a more influential factor in the communication difficulties diagnostic of autism, as opposed to a "deficit" in understanding social meanings. If this claim is borne out, it would have a couple of implications for psychological practice. First, it would suggest that interventions targeting IU may be useful for autistic people wanting support with communication challenges. Current interventions for communication focus on explicit 
129 instruction in social skills, and reviews suggest modest effectiveness although there are questions

130 about the extent to which skills transfer to daily life (Gates, Kang, \& Lerner, 2017; Spain \&

131 Blainey, 2015). A focus on IU may be a useful alternative target. Existing cognitive interventions

132 involve integrating psychoeducation and cognitive challenge techniques to target a client's

133 beliefs about (un)certainty, and these have shown some effectiveness for treating mental health

134 difficulties and particularly anxiety in the general population (Shihata et al., 2016). It remains to

135 be seen whether such interventions could be adapted to support autistic individuals with

136 distressing communication experiences, although this is a promising possibility given that early

137 studies suggest that such interventions may be feasible and acceptable for autistic groups

138 (Rodgers et al., 2018; Rodgers et al., 2016). Second, if a cognitive preference for certainty is

139 especially significant as an explanation for social difficulties, then it supports an autism-positive

140 approach to intervention which focuses on awareness of cognitive differences across

141 communities. In addition, if performance on the Implicature Comprehension Test is a sensitive

142 marker of IU, that in itself might have clinical and research utility, since measurement of IU is

143 currently limited to self- and informant-report questionnaires.

A remaining question is whether any differences observed on our tasks are specific to

autism or might also be relevant to other neurodevelopmental diagnoses. This is certainly

plausible in the light of dimensional models of neurodiversity, where features of autism,

147 developmental language disorder, dyslexia, ADHD, etc., might show some overlap and exist as a

148 continuum in the general population (Thapar, Cooper, \& Rutter, 2017). To test the specificity of

149 any cognitive differences observed on our tests, we will compare performance by autistic people

150 to both a dyslexic and a general population sample. As neurodevelopmental conditions are often

151 co-occurring, we view these three groups as defined less by a specific diagnostic label but rather 
152 as varying along a communication continuum. As such, one group is defined by social

153 communication differences potentially alongside co-occurring language/literacy impairments

154 (the autism group), a second group by language/literacy impairments but no diagnosed social

155 communication difficulties (the dyslexia group), and a final group without any communication,

156 language or literacy related diagnosis. It is possible that dyslexic adults may show some

157 difficulty on our pragmatic task (i.e. the Implicature Comprehension Test), as previous research

158 has documented some limited evidence for pragmatic difficulties in dyslexic individuals (e.g.

159 Cappelli et al., 2018; Cardillo et al., 2018; Griffiths, 2007). An alternative possibility is that

160 adults with dyslexia will show greater difficulty with tasks focused more on structural language

161 skills compared to pragmatics. For instance, a meta-analysis has found that dyslexic adults

162 perform less well on language measures, such as vocabulary, speeded naming, verbal memory

163 and phonological processing, than people without a diagnosis of dyslexia, with moderate to large

164 effect sizes (Swanson \& Hsieh, 2009). Given that there is no clear reason to support one of these

165 possibilities over the other, we will take a more exploratory approach with the dyslexic group to

166 examine how they compare with autistic adults.

In summary, we propose the following hypotheses:

168
(1) Autistic adults will score lower on a pragmatic language task when responses are coded purely in terms of confidence (number of yes and no responses, regardless of polarity) than when responses are coded in terms of accuracy (with yes and maybe yes, and maybe no and no responses, combined according to polarity), compared to adults without any neurodevelopmental diagnosis, but will not show this same disparity between accuracy and confidence on a core language task. 
(2) The number of less confident responses (maybe responses) on the pragmatic language task, the score on the Intolerance of Uncertainty Scale, and selfreported social communication difficulties will significantly intercorrelate across the full sample.

\section{Methods}

Ethical approval for this project was granted on 30/03/2020 by the Medical Science Interdivisional Research Ethics Committee at Oxford University (Ref: R68518/RE001). The script for the power analysis and example materials are available on the Open Science

Framework here: https://osf.io/wk97s/. We are very happy to share full materials for this study, but to protect the validity of the items for future uses, we ask that researchers contact us to request a link to the full assessments. See further information on requesting access by following the link above.

\section{Power calculation}

We determined power to detect the three-way interaction described in Hypothesis 1 using simulations. We used data reported in Wilson and Bishop (2020b) to estimate the likely size of fixed and random effects in the mixed model described in Data Analysis below. Using R package simr (Green \& MacLeod, 2016) we ran 1000 simulations with a sample size of 200 people (100 autistic, 100 non-autistic) and a significant three-way interaction was found in 9830 simulations, indicating that power was over $98 \%$ to detect our effect of interest at an alpha level of .05 . Effectively, this allows us to detect a significant difference where approximate Cohen's $d$ values 
194 in favour of the non-autistic group are 0.70 and 1.10 for the implicature accuracy and confidence

195 variables and 0.20 for the grammar variables, as suggested by our previous data. Allowing for

196 exclusion of up to $10 \%$ of participants during the outlier exclusion phase described in Data

197 Analysis, power remains very high (98\% in a sample of 180). For hypothesis 2, a sample of 200

198 is powered at over $99 \%$ to detect a correlation of .3 .

\section{Participants}

We will recruit individuals with autism, dyslexia, and no neurodevelopmental diagnosis.

201

202

203

204

205

206

207

208

209

210

211

212

213

214

215

Based on the power calculation, we will recruit 100 autistic adults and 100 adults without a neurodevelopmental diagnosis in order to run the confirmatory analysis. In addition, we will aim to recruit 50 dyslexic adults as a clinical control group for exploratory analysis. All participants will meet the following eligibility criteria: (i) age of 18 years or over, (ii) native-level fluency in English, (iii) no history of acquired brain injury, (iv) no significant uncorrected sensory impairment, and (v) access to a computer with internet and audio.

Individuals will be recruited into three groups defined by communication and language/literacy problems. One group will be recruited on the basis of a clinical diagnosis of autism; participants will need to declare where, by whom and what label was used for their diagnosis on the Study Questionnaire. For inclusion, the diagnosis must have been made in a clinical service by appropriately trained individuals, such as clinical psychologists, psychiatrists or developmental paediatricians. We will recruit autistic individuals through Autistica, the research network for families and individuals with autism, as well as support groups arranged privately and by the National Autistic Society, and through social media. A second group will include individuals reporting dyslexia or specific reading difficulties. For inclusion in this group, 
216 individuals must score below the clinical threshold of 6 on the Autism Spectrum Quotient (AQ)

217 and at 6 or above on the Reading Scale of the Adult Reading Questionnaire (ARQ); this latter

218 score translates to over 1.5 SDs above the mean in individuals not self-reporting dyslexia in the

219 original validation study (Snowling, Dawes, Nash, \& Hulme, 2012). Individuals will be recruited

220 through charitable organisations such as the British Dyslexia Association and social media.

221 Other neurodevelopmental diagnoses will be noted but will not be grounds for exclusion from

222 these groups. A third group will have no neurodevelopmental diagnosis, and will be recruited via

223 the online participant platform, Prolific (https://prolific.co). Individuals will be excluded from

224 this third group if they score above threshold on either the AQ or ARQ (i.e. above 6 on either)

225 and if they have ever been diagnosed with: a global or specific learning disability, attention

226 deficit hyperactivity disorder, dyspraxia/developmental coordination disorder, a genetic variation

227 (such as Down's syndrome or Fragile-X) or a neurological condition (such as epilepsy).

\section{Procedure}

229 The study will be presented online using Gorilla, the online platform for behavioural

230 experiments and surveys (https://gorilla.sc/). Individuals complete an online set of tasks and

231 questionnaires in one sitting at a time and place of their choosing. After providing informed

232 written consent to participate, individuals will complete a Study Questionnaire (please see the

233 OSF link) on which they will be asked to report on demographics and any neurodevelopmental

234 diagnoses. Then they will complete questionnaires/tasks in two sections. The first section will

235 include the experimental tasks required for the hypothesis-testing, and the second will include

236 several brief measures for characterising the sample. The two experimental tasks will be

237 randomized between participants, and all other measures will be administered in the order set out

238 below. 
Section 1: Experimental tasks assessing ability and confidence with pragmatics and core

Implicature Comprehension Test-2 (ICT-2). In this test of pragmatic language comprehension, participants complete an adapted version of the Implicature Comprehension Test (Wilson \& Bishop, 2019). There is a sequence of 56 videos, each approximately $8 \mathrm{~s}$ long, consisting of a conversational adjacency pair between two characters: the first character asks a closed question (eliciting a "yes" or "no" answer) and the second character produces a short answer but does not say yes or no. Each utterance is between 5 and 10 words in length, grammatically simple, and age of acquisition of the words does not exceed middle primary school level. Following the dialogue, the participant hears a comprehension question directly based on the structure of the first character's question. The participant answers the question on a 4-point scale (yes, maybe yes, maybe no, no) by clicking buttons arranged horizontally on the screen. This is a timed task, with a time limit of $10 \mathrm{~s}$ for a response from the offset of the question. There are two item types: implicature and explicit-response. Utterance length and psycholinguistic variables (word frequency, word age-of-acquisition and word concreteness) are controlled across the two item types.

For 40 videos, the second character's answer is indirect, and the participant needs to process implicature to answer the comprehension question appropriately. Example:

Character 2: There were lots of trains going past. 
Answer: No

Half of the comprehension questions are correctly answered by "yes" and half by "no".

263

264

265

266

267

268

269

270

271

272

273

274

275

276

277

278

279

280

281

282

Alongside the implicature items, there are 16 explicit-response items where the second character's answer is more explicit. In these items, the speaker intends to convey uncertainty explicitly, whereas in the implicature items, the uncertainty is in the mind of the listener.

Example:

Character 1: Will we get there by seven?

Character 2: Mmm, yes maybe, I think we're near.

Comprehension Question: Will they get there by seven?

Answer: Maybe yes

For these items, the comprehension questions will encourage the participant to use the full scale, with four questions each correctly answered by "yes", "maybe yes", "maybe no" and "no". There is one measured variable: total accuracy out of 16 .

Grammaticality Decision Test (GDT; based on Wilson \& Bishop, 2019). In this test of core language ability, participants listen to a sequence of 50 sentences and decide if the sentence is grammatical and well-formed or not. Half the sentences are grammatical. Grammatical violations represent mistakes that native speakers would not tend to make, such as using an incorrect verb form (e.g. I went out after I have eaten dinner) or atypical placing of adverbs (e.g. If you can't find it, I can send again the letter). Participants are asked whether the sentences are 
283 grammatical, indicating "yes", "maybe yes", "maybe no" and "no" as their answer by clicking

284 buttons arranged horizontally on the screen, as in the ICT-2. After offset of the sentence,

285 participants have 10 seconds to give their response.

286 Section 2: Questionnaires and tasks for characterising the sample

Autism Spectrum Quotient-10 (AQ-10; Allison, Auyeung, \& Baron-Cohen, 2012).

Autistic traits will be measured using this 10-item version of the Autism Spectrum Quotient

(AQ). In the original validation study, the measure had $85 \%$ correct discrimination between almost 450 autistic adults and over 800 control adults. The National Institute for Health and Care Excellence (NICE, 2012) recommend use of the questionnaire for identifying individuals for comprehensive autism assessment. A clinical cut-off of 6 or more is taken as indicating possible autism.

This is a norm-referenced questionnaire measuring self-reported communication challenges.

Participants will be presented with the pragmatic language scale (22 items). For each item, participants identify how frequently certain communication behaviours apply to them on a 4point scale from "less than once a week (or never)" to "several times a day (or all the time)". An example item is "People tell me that I ask the same question over and over".

Hulme, 2012). Self-reported reading difficulties will be measured using this 5 -item observed literacy ability at -.67) and, along with self-reported dyslexia status, discriminated with $88 \%$ accuracy in identifying those with weaker literacy skills. 
306

307

308

309

310

311

2014). This is an open-access test of general cognitive ability, which requires participants to complete 16 items across four item types: matrix reasoning, verbal reasoning, three-dimensional rotation, and letter-number sequences. The ICAR sample test has good internal consistency $($ alpha $=.81)$, and good convergent validity (correlating at approximately .8 with commercial IQ measures when correcting for reliability and restriction of range; Condon \& Revelle, 2014; Young \& Keith, 2020).

Synonyms Test (Wilson, \& Bishop, 2019). General verbal ability will be measured using this 25-item test of vocabulary knowledge. Participants select which of five written words is synonymous with a target word, under a 12-second time limit. The original version of the GDT and this task showed a moderate correlation in both autistic and non-autistic samples, suggesting they are overlapping measures of core language ability (Wilson \& Bishop, 2019, 2020a).

Intolerance of Uncertainty Scale (IUS-12; Carleton, Norton, \& Asmundson, 2007). In this self-report measure of intolerance of uncertainty, participants are presented with 12 statements about uncertainty, ambiguous situations, and the future. They rate how closely each statement relates to them on a 5-point scale from "not at all characteristic of me" to "entirely characteristic of me". An example item is: "When I am uncertain, I can't function very well."

\section{Data Analysis}

Individuals will be excluded from the dataset if they have an outlying score for either (a) accuracy on the GDT or the positive control items of the ICT-2 (b) total number of timeouts across the ICT-2 and GDT. Outliers will be defined according to the method of Hoaglin and Inglewicz (1987): more than 2.2 times the interquartile range below the first quartile. In previous 
327 work, these criteria led to exclusion of approximately 5\% of participants, and captured

328 individuals scoring below approximately $50 \%$ on the GDT and $70 \%$ on the positive control items

329 of the original version of the ICT (Wilson \& Bishop, 2020a).

Data will be analysed in R (R Core Team, 2019). After exclusions, total scores on the two experimental tasks for the groups with autism and no neurodevelopmental diagnosis will be turned into long format, and each participant's total will be coded for task (ICT-2 or GDT), group (autistic or no neurodevelopmental diagnosis), response (accuracy or confidence) and participant. We will run a mixed effects linear regression using the lme4 $\mathrm{R}$ package (Bates, Maechler, Bolker, \& Walker, 2015). The model will include three fixed effects (task, group and response) and the interactions between these, as well as a random effect (participant). The significance level of the three-way interaction will offer a test of Hypothesis 1 . We will also compute correlations between confidence on the ICT-2, self-reported communication challenges on the CC-SR and total score on IUS-12 across the full sample; this will test Hypothesis 2 and represents a dimensional analysis of the relationship between communication difficulties and sensitivity to uncertainty. Table 1 shows a summary of our planned analyses, linking research questions, hypotheses, tests and power calculations. group and the group without a neurodevelopmental diagnosis on the ICT-2 and GDT in terms of accuracy and confidence.

\section{References}

Allison, C., Auyeung, B., \& Baron-Cohen, S. (2012). Toward brief "Red Flags" for autism screening: The Short Autism Spectrum Quotient and the Short Quantitative Checklist for 
Autism in toddlers in 1,000 cases and 3,000 controls [corrected]. Journal of the American Academy of Child and Adolescent Psychiatry, 51(2), 202-212. doi:10.1016/j.jaac.2011.11.003

American Psychiatric Association. (2013). Diagnostic and statistical manual of mental disorders (5th ed.). Arlington, VA: American Psychiatric Publishing.

Baird, G., \& Norbury, C. F. (2016). Social (pragmatic) communication disorders and autism spectrum disorder. Archives of Disease in Childhood, 101(8), 745-751. doi:10.1136/archdischild-2014-306944

Baron-Cohen, S. (1988). Social and pragmatic deficits in autism: Cognitive or affective? Journal of Autism and Developmental Disorders, 18(3), 379-402. doi:10.1007/BF02212194

Bates, D., Maechler, M., Bolker, B., \& Walker, S. (2015). Fitting linear mixed-effects models using lme4. Journal of Statistical Software, 67(1), 1-48. doi:10.18637/jss.v067.i01

Baxter, D. M., \& Warrington, E. K. (1994). Measuring dysgraphia: A graded-difficulty spelling test. Behavioral Neurology, 7(3), 107-116. doi:10.3233/BEN-1994-73-401

Bishop, D. V. M., Whitehouse, A. J. O., \& Sharp, M. (2009). Communication Checklist - Self Report (CC-SR). Pearson.

Cappelli, G., Noccetti, S., Arcara, G., \& Bambini, V. (2018). Pragmatic competence and its relationship with the linguistic and cognitive profile of young adults with dyslexia. Dyslexia, 24(3), 294-306. doi:10.1002/dys.1588 
368 Cardillo, R., Garcia, R., B., Mammarella, I., C., \& Cornoldi, C. (2018). Pragmatics of language

369 and theory of mind in children with dyslexia with associated language difficulties or nonverbal learning disabilities. Applied Neuropsychology Child, 7(3), 245-256. doi:10.1080/21622965.2017.1297946

Condon, D. M., \& Revelle, W. (2014). The International Cognitive Ability Resource: Development and initial validation of a public-domain measure. Intelligence, 43, 52-64.

Frith, U. (1989). Autism: Explaining the enigma. Blackwell.

Gates, J. A., Kang, E., \& Lerner, M. D. (2017). Efficacy of group social skills interventions for youth with autism spectrum disorder: A systematic review and meta-analysis. Clinical Psychology Review, 52, 164-181. https://doi.org/10.1016/j.cpr.2017.01.006 mixed models by simulation. Methods in Ecology and Evolution, 7(4), 493-498. doi:10.1111/2041-210X.12504 
389 Griffiths, C. C. (2007). Pragmatic abilities in adults with and without dyslexia: a pilot study.

$390 \quad$ Dyslexia, 13(4), 276-296. doi:10.1002/dys.333

391 Hoaglin, D. C., \& Iglewicz, B. (1987). Fine-tuning some resistant rules for outlier labeling.

392

393

394

395

396

397

398

399

400

401

402

403

404

405

406

407

408

409

Journal of the American Statistical Association, 82(400), 1147-1149.

doi:10.1080/01621459.1987.10478551

Hobson, R. P. (2012). Autism, literal language and Concrete thinking: Some developmental considerations. Metaphor and Symbol, 27(1), 4-21. doi:10.1080/10926488.2012.638814

Jenkinson, R., Milne, E., \& Thompson, A. (2020). The relationship between intolerance of uncertainty and anxiety in autism: A systematic literature review and metaanalysis. Autism. https://doi.org/10.1177/1362361320932437

Kalandadze, T., Norbury, C. F., Nærland, T., \& Næss, K. B. (2018). Figurative language comprehension in individuals with autism spectrum disorder: A meta-analytic review. Autism, 22(2), 99-117. doi:10.1177/1362361316668652

Loukusa, S., \& Moilanen, I. (2009). Pragmatic inference abilities in individuals with Asperger syndrome or high-functioning autism. A review. Research in Autism Spectrum Disorders, 3(4), 890-904. doi:10.1016/j.rasd.2009.05.002

Maras, K., Norris, J. E., \& Brewer, N. (2020). Metacognitive monitoring and control of eyewitness memory reports in autism. Autism Research. https://doi.org/10.1002/aur.2278

National Institute for Health and Care Excellence (2012). Autism spectrum disorder in adults: diagnosis and management (Clinical guideline CG142). Retrieved from https://www.nice.org.uk/guidance/cg142 
410 Nicholson, T., Williams, D. M., Grainger, C., Lind, S. E., \& Carruthers, P. (2019). Relationships

411

412

413

414

415

416

417

418

419

420

421

422

423

424

425

426

427

428

429 between implicit and explicit uncertainty monitoring and mindreading: Evidence from autism spectrum disorder. Consciousness and Cognition, 70, $11-24$. https://doi.org/10.1016/j.concog.2019.01.013

R Core Team (2019). R: A language and environment for statistical computing. R Foundation for Statistical Computing, Vienna, Austria. URL:https://www.R-project.org/

Rapin, I., \& Dunn, M. (2003). Update on the language disorders of individuals on the autistic spectrum. Brain and Development, 25(3), 166-172. doi:10.1016/S0387-7604(02)00191-2

Rodgers, J., Herrema, R., Honey, E., \& Freeston, M. (2018). Towards a treatment for intolerance of uncertainty for autistic adults: A single case experimental design study. Journal of Autism and Developmental Disorders, 48(8), 2832-2845. https://doi.org/10.1007/s10803018-3550-9

Rodgers, J., Hodgson, A., Shields, K., Wright, C., Honey, E., \& Freeston, M. (2017). Towards a treatment for intolerance of uncertainty in young people with autism spectrum disorder: Development of the Coping with Uncertainty in Everyday Situations (CUESC) programme. Journal of Autism and Developmental Disorders, 47(12), 3959-3966. https://doi.org/10.1007/s10803-016-2924-0

Shihata, S., McEvoy, P. M., Mullan, B. A., \& Carleton, R. N. (2016). Intolerance of uncertainty in emotional disorders: What uncertainties remain? Journal of Anxiety Disorders, 41, 115-124. https://doi.org/10.1016/j.janxdis.2016.05.001 
430 Snowling, M., Dawes, P., Nash, H., \& Hulme, C. (2012). Validity of a Protocol for Adult

431

432

433

434

435

436

437

438

439

440

441

442

443

444

445

446

447

448

Self-Report of Dyslexia and Related Difficulties. Dyslexia, 18(1), 1-15. doi:10.1002/dys. 1432

Spain, D., \& Blainey, S. H. (2015). Group social skills interventions for adults with highfunctioning autism spectrum disorders: A systematic review. Autism, 19(7), 874-886. https://doi.org/10.1177/1362361315587659

Sperber, D., \& Wilson, D. (1986). Relevance: Communication and cognition. Blackwell.

Swanson, H. L., \& Hsieh, C. J. (2009). Reading disabilities in adults: A selective meta-analysis of the literature. Review of Educational Research, 79(4), 1362-1390. doi:10.3102/0034654309350931

Thapar, A., Cooper, M., \& Rutter, M. (2017). Neurodevelopmental disorders. Lancet Psychiatry, 4(4), 339-346. doi:10.1016/S2215-0366(16)30376-5

Wakabayashi, A., Baron-Cohen, S., Wheelwright, S., Goldenfeld, N., Delaney, J., Fine, D., Smith, R., \& Weil, L. (2006). Development of short forms of the Empathy Quotient (EQShort) and the Systemizing Quotient (SQ-Short). Personality and Individual Differences, 41(5), 929-940. doi:10.1016/j.paid.2006.03.017

Wilson, A., C., \& Bishop, D., V., M., (2019). "If you catch my drift...": Ability to infer implied meaning is distinct from vocabulary and grammar skills [version 3; peer review: 3 approved]. Wellcome Open Research, 4(68). doi:10.12688/wellcomeopenres.15210.3 
449 Wilson, A. C., \& Bishop, D. V. M. (2020a). Judging meaning: A domain-level difference $450 \quad$ between autistic and non-autistic adults. Royal Society Open Science (In press)

451 Wilson, A. C., \& Bishop, D. V. M. (2020b). "Second guessing yourself all the time about what 452 they really mean...": Cognitive differences between autistic and non-autistic adults in 453 understanding implied meaning. Autism Research. https://doi.org/10.1002/aur.2345

454 Young, S. R., \& Keith, T. Z. (2020). An examination of the convergent validity of the ICAR16 455 and WAIS-IV. Journal of Psychoeducational Assessment.

456 https://doi.org/10.1177/0734282920943455 
Table $\mathbf{1}$ (on next page)

Planned Analyses. 


\begin{tabular}{|c|c|c|c|}
\hline Research Question & Hypothesis & Statistical Analysis & Power Analysis \\
\hline $\begin{array}{l}\text { Do autistic people show } \\
\text { reduced confidence in } \\
\text { understanding implied } \\
\text { meanings in conversation? }\end{array}$ & $\begin{array}{l}\text { Autistic adults will score lower } \\
\text { on the Implicature } \\
\text { Comprehension Test- } 2 \text { when } \\
\text { responses are coded in terms of } \\
\text { confidence (number of yes and } \\
\text { no responses, regardless of } \\
\text { polarity) than when responses } \\
\text { are coded in terms of accuracy } \\
\text { (with yes and maybe yes, and } \\
\text { maybe no and no responses, } \\
\text { combined according to } \\
\text { polarity), compared to adults } \\
\text { without any } \\
\text { neurodevelopmental diagnosis, } \\
\text { but will not show this same } \\
\text { disparity between accuracy and } \\
\text { confidence on the } \\
\text { Grammaticality Decision Test. }\end{array}$ & $\begin{array}{l}\text { A mixed model will be run } \\
\text { including the following effects: } \\
\text { task (Implicature } \\
\text { Comprehension Test-2 or } \\
\text { Grammaticality Decision Test), } \\
\text { group (autistic or no } \\
\text { neurodevelopmental } \\
\text { diagnosis), and response } \\
\text { (confidence or accuracy) as } \\
\text { fixed effects; the interactions } \\
\text { between these fixed effects; } \\
\text { and participant as a random } \\
\text { effect. The significance level of } \\
\text { the three-way interaction will } \\
\text { offer a test of the hypothesis. }\end{array}$ & $\begin{array}{l}\text { A sample of } 200 \text { people is } \\
\text { powered at over } 98 \% \text { to detect } \\
\text { the three-way interaction. }\end{array}$ \\
\hline $\begin{array}{l}\text { Do individual differences in } \\
\text { confidence in interpreting } \\
\text { meaning, intolerance of } \\
\text { uncertainty and self-rated } \\
\text { communication difficulties } \\
\text { inter-correlate? }\end{array}$ & $\begin{array}{l}\text { The number of less confident } \\
\text { responses (maybe responses) } \\
\text { on the Implicature } \\
\text { Comprehension Test-2, the } \\
\text { score on the Intolerance of } \\
\text { Uncertainty Scale, and self- } \\
\text { reported social communication } \\
\text { difficulties on the CC-SR will } \\
\text { significantly intercorrelate } \\
\text { across the full sample. }\end{array}$ & $\begin{array}{l}\text { Pearson's correlations will be } \\
\text { computed to quantify the } \\
\text { relationships between these } \\
\text { three variables across the } \\
\text { whole sample. }\end{array}$ & $\begin{array}{l}\text { A sample of } 200 \text { people is } \\
\text { powered at over } 99 \% \text { to detect } \\
\text { correlations of } .3 \text {. }\end{array}$ \\
\hline
\end{tabular}

